\title{
Improvement of Crystal Quality of a Homoepitaxially Grown Diamond Layer Using Plasma Etching Treatment for a Diamond Substrate
}

\author{
Yuta KONNO $^{1 *}$, Junichi H. KANEKO ${ }^{1}$, Fumiyuki FUJTTA ${ }^{1}$, Hideyuki WATANABE ${ }^{2}$, Kenji HARA ${ }^{3}$, Kei SATO ${ }^{1}$ \\ Akinori KAKIMOTO ${ }^{1}$, Shinichi SHIKATA ${ }^{2}$, Akira HOMMA ${ }^{1}$, Michihiro FURASAKA ${ }^{1}$ \\ ${ }^{I}$ Graduate school of Engineering, Hokkaido University, N13, W8 , Kita-ku, Sapporo 060-8628, Japan \\ ${ }^{2}$ AIST Tsukuba Central , 21-1-1 Umezono, Tsukuba, Ibaraki 305-8568, Japan \\ 3 Graduate school of Science, Hokkaido University, N10, W8, Sapporo 060-0810, Japan
}

\begin{abstract}
To realize a practical application of synthetic diamond radiation detectors, an effort to synthesize high-quality diamond single crystals was conducted using microwave plasma assisted chemical vapor deposition (CVD). This study evaluated the influence of etching treatment - a reactive ion-etching technique used for diamond substrates - on the crystal quality of a homoepitaxially grown diamond layer. A type $\mathrm{Ib}$ diamond single crystal grown using a high-temperature and high-pressure method was used as the substrate. Homoepitaxial diamond layers with ca. $10 \mu \mathrm{m}$ thickness were grown on diamond substrates with different etching depths. Then observations were conducted using a differential interference microscopy and cathode luminescence spectroscopy. Judging from the intensity of free exciton recombination luminescence and other observations, the crystal quality was markedly improved by the etching treatment on the diamond substrates. Furthermore, a macroscopic complex of abnormal growth resulting from a mechanical polished flaw was almost removed by the etching treatment with etching depth of $2.6 \mu \mathrm{m}$.
\end{abstract}

KEYWORDS: detector, chemical vapor deposition diamond, reactive ion etching, cathode luminescence

\section{Introduction ${ }^{1}$}

Diamond presents several merits of high-temperature operation ${ }^{1)}$, corrosion tolerance ${ }^{2)}$, radiation resistance ${ }^{3)}$, etc. Therefore, practical diamond electric devices including sensor application are desired in fields of nuclear engineering, radiology, and space engineering. In addition, diamond is applicable to a $14 \mathrm{MeV}$ neutron energy spectrometer for ion temperature measurements using ${ }^{12} \mathrm{C}(\mathrm{n}, \alpha){ }^{9} \mathrm{Be}$ reaction ${ }^{4-5)}$. The final goal of our research is realization of a practical synthetic diamond radiation detector to achieve that purpose.

A homoepitaxial CVD diamond single crystal has been grown on a (001) surface of a high-temperature and high-pressure (HP/HT) type Ib diamond single crystal substrate. To realize a practical synthetic diamond radiation detector, reduction of the loss of charge carriers in a diamond crystal is indispensable. Therefore, impurities and defects in a crystal must be removed to the greatest degree possible. A $\mathrm{HP} / \mathrm{HT}$ type Ib diamond single crystal substrate had an optically smooth surface fabricated by mechanical polishing. The crystallinity near the substrate surface deteriorated by this process and the influence on homoepitaxial growth was expected. Nevertheless, it is known that the damaged layer near the surface was removed by etching ${ }^{6}$.

This study evaluated effects of reactive ion etching treatment of a $\mathrm{HP} / \mathrm{HT}$ diamond substrate on the crystalline quality of a homoepitaxially grown diamond layer.

\section{Experimental}

Conventional wet etching process suites used for silicon and metals are difficult to apply to diamond because the latter

*Corresponding Author, Y.Konno@eng.hokudai.ac.jp

(C) Atomic Energy Society of Japan material is very physically and chemically stable. N.N. Efremow et al. reported ion beam etching using $\mathrm{Xe}+$ ions; their equipment was complex and the process needed additional treatment for obtaining a flat surface. In general speaking, ion etching shows strong anisotropy ${ }^{7-8)}$.

Consequently, etching processes using plasma or ion beams are generally used ${ }^{9)}$. For this study, high-frequency ion plasma etching - a reactive ion etching (RIE) technique - was adopted. An RIE apparatus (RIE-10NRV; Samco, Inc.) was used to etch a (001) surface of a HP/HT type Ib diamond single crystal grown by Sumitomo Electric Industries, Ltd.

Etching conditions and results are presented in Table 1. A mixture of $\mathrm{CF}_{4}$ and $\mathrm{O}_{2}$, as $\mathrm{CF}_{4} / \mathrm{O}_{2}: 20 \%$, was used as the processing gas ${ }^{10}$. For the individual use of $\mathrm{O}_{2}$ gas, a metallic material that is a constituent of a reactor chamber adheres on a substrate and then disturbs etching. That is designated as a metal mask effect ${ }^{11)}$. To suppress this effect, $\mathrm{CF}_{4}$ gas was adopted for use as a processing gas component. Surfaces of the substrates before etching were optically smooth; only polishing flaws with depth of tens of nanometers were observed using atomic force microscopy (AFM, JSPM5200; JEOL).

To evaluate effects of the etching process for the substrate on quality of a homoepitaxially grown diamond, a half plane of a substrate was masked by aluminum. The aluminum mask thickness was ca. $0.5 \mu \mathrm{m}$. It was fabricated using an evaporation method. The etching rate of aluminum is six times lower than that of diamond ${ }^{12}$. After etching, the aluminum mask was removed by polishing using $\mathrm{Al}_{2} \mathrm{O}_{3}$ abrasive soap. Subsequently, the etched depth-the difference in height between the etched and non-etched surfaces - was measured using a stylus method. After the 
etching process, a homoepitaxial diamond layer whose thickness was ca. $10 \mu \mathrm{m}$ was grown on the HP/HT type Ib diamond substrate using a microwave plasma assisted chemical vapor deposition apparatus (AX5250; Seki Technotron Corp.). Growth conditions were the following: total pressure of $\mathrm{H}_{2}$ and $\mathrm{CH}_{4}, 110$ Torr; concentration of $\mathrm{CH}_{4}$, $4 \%$; temperature of a substrate, $800^{\circ} \mathrm{C}$. To facilitate verification of the effect of the etching process, growth conditions in which abnormal grain growth tended to occur were adopted.

After crystal growth, surface characteristics were observed using a differential interference microscope; the crystalline quality was evaluated using $\mathrm{CL}$ spectroscopy. The CL spectra were measured using the following conditions: sample temperature, $79 \mathrm{~K}$; electron beam accelerating voltage, $13 \mathrm{kV}$; electron beam current, $50 \mathrm{nA}$; observation area, ca. $190 \times 190 \mu \mathrm{m}$; diffraction grating, 300/mm; detector, a CCD camera.

Table1 Etching condition and results

\begin{tabular}{|c||c|c|c|}
\hline Sample ID & 01 & 02 & 03 \\
\hline RF Power $(\mathrm{W})$ & \multicolumn{3}{|c|}{200} \\
\hline $\mathrm{O}_{2}$ gas flow rate $(\mathrm{sccm})$ & \multicolumn{3}{|c|}{16} \\
\hline $\mathrm{CF}_{4}$ gas flow rate $(\mathrm{sccm})$ & \multicolumn{3}{|c|}{4} \\
\hline $\mathrm{CF}_{4} / \mathrm{O}_{2}(\%)$ & \multicolumn{3}{|c|}{20} \\
\hline Total gas pressure $(\mathrm{Pa})$ & \multicolumn{3}{|c|}{5} \\
\hline Etching time $(\mathrm{hr})$ & 2.5 & 2.5 & 4.7 \\
\hline Etching depth $(\mu \mathrm{m})$ & 1.0 & 1.6 & 2.6 \\
\hline Etching rate $(\mu \mathrm{m} / \mathrm{h})$ & 0.41 & 0.64 & 0.55 \\
\hline
\end{tabular}

\section{Results and discussion}

Figure 1 portrays differential interference images of three diamond samples with different etching depths. Lines located at the center of each sample were cliffs created by the etching. For samples $\# 01, \# 02, \# 03$, the left side parts from these lines were etched, respectively, to 1.0, 1.6 and $2.6 \mu \mathrm{m}$ depths. For right side parts, surfaces were not etched. In Fig. 1, circles show locations at which CL spectroscopy were conducted: (a) shows a flat part on the etched side, (b) is a hillock part on the etched side, and (c) is a flat part on the non-etched side. Although these samples were synthesized under the same growth conditions, large differences in morphology were observed. Individual differences in substrates strongly influenced the homoepitaxial diamond morphology. As described herein, influences on results arising from individual differences in substrates were ruled out to the greatest degree possible.

For sample $\# 01$, with an etching depth of $1.0 \mu \mathrm{m}$, a continuous complex of abnormal growth, which is expected to result from the influence of polishing flaws, traverses the centerline, as presented in Fig. 1 A) at (d). On the other hand, for sample $\# 03$, with an etching depth of $2.6 \mu \mathrm{m}$, as presented in Fig. 1 C), a similar continuous complex almost disappears on the left side: the etched side. Based on this result, an etching depth of ca. $3 \mu \mathrm{m}$ is sufficient to remove complexes of abnormal growth for a HP/HT type Ib diamond single crystal substrates used for this study.

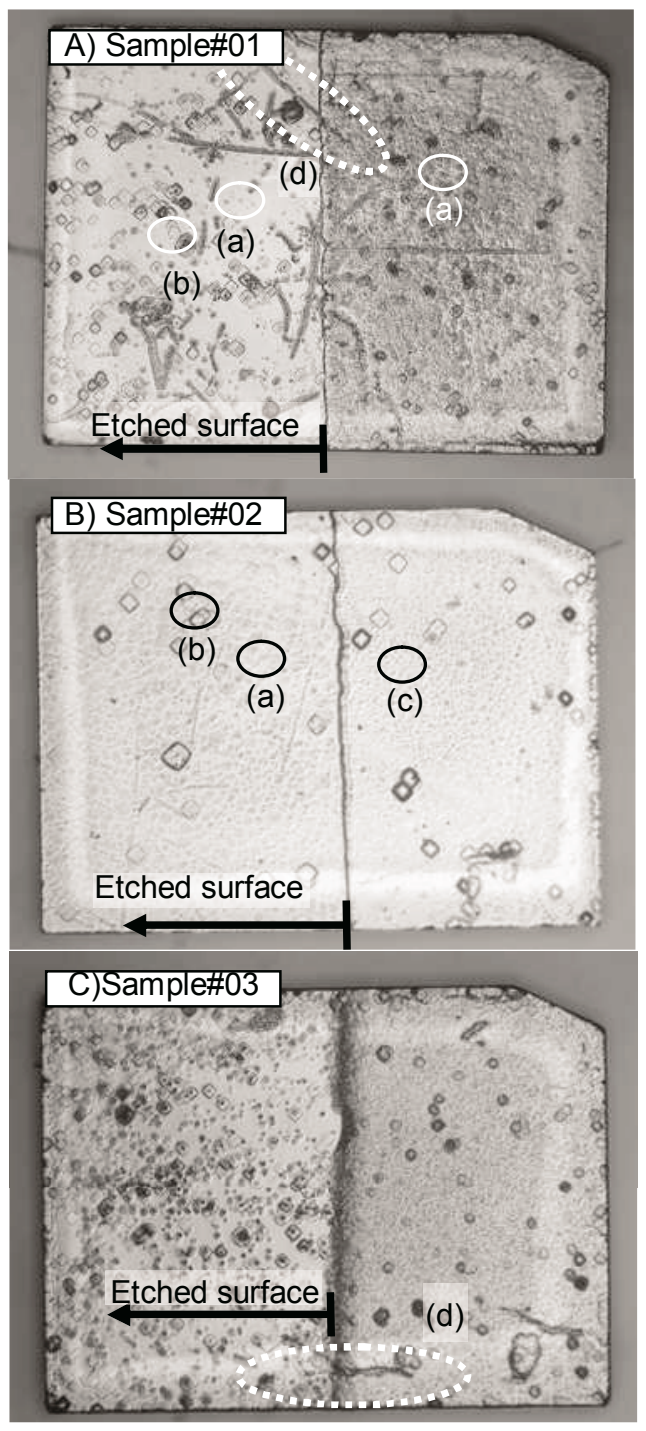

Fig.1 Differential interference microscopic images of diamond samples after etching and homoepitaxial diamond growth: A) Sample \#01, B) Sample \#02, and C) Sample \#03. Lines located at the center of each sample are cliffs caused by etching. The left side part from this line was etched 1.0, 1.6, and $2.6 \mu \mathrm{m}$ deep using the RIE method. The right side part has no etching. (a), (b), (c) show points where cathode luminescence spectra measurements were conducted.

Figure 1 C) depicts sample $\# 03$, it is readily apparent that densities of abnormal growth and/or hillocks were greater on the etched side than on the non-etched sides. A possible explanation of this tendency is increased roughness by etching. For sample $\# 02$, the average roughness of the surfaces was increased from 1.532 to $3.589 \mathrm{~nm}$ during the etching process. Although changes on the average roughness of the surface for sample \#01 and 03 could not be measured, the same tendency was confirmed using other substrates. These values were measured using AFM. However, no marked difference of densities of abnormal growth or 
hillocks was found. Unfortunately, no data of average roughness were obtained before and after the etching process for sample \#03. Therefore, it is impossible to specify the relation between roughness and density of abnormal growth or hillocks at present. Further investigation, including measurement of shape and size of abnormal growth and hillocks, of the relation between roughness of a substrate surface and densities of abnormal growth and/or hillocks is necessary.

Cathode luminescence spectra obtained from samples \#01 and $\# 02$ are presented in Fig. 2 and Fig. 3. For easy of comprehension, each spectrum measured at the indicated point on the same sample is displayed with a shifted vertical axis. For sample \#01, large differences in the intensity of free exciton recombination (FE) luminescence located at 235 $\mathrm{nm}$ were observed, respectively, between the flat parts on (a) the etched and (c) the non-etched part, 1070 and 270 counts. For sample \#02, a more remarkable difference was observed in the yield of FE luminescence: 815 and 27 counts for the flat parts on the etched and non-etched part. Free exciton recombination luminescence reflects the width of band gap energy. The FE luminescence is only visible for a high-quality diamond; its intensity decreases drastically with the presence of impurities or defects ${ }^{13-14)}$. Judging from these results, the etching process on the HP/HT type Ib diamond single crystal substrate improved the crystalline quality.

The same tendency was confirmed for other spectral areas. Band 'A', with its peak located at around 440-460, is known for its relation with structural defects. Figures 2(a) and 2(c) show the same CL spectra as those in Fig. 4 with an expanded vertical axis. Band ' $\mathrm{A}$ ' luminescence was also lower in the flat area of the etched part than in the non-etched part. The same result was observed for sample \#2. The etching process on the substrates decreased structural defects in the homoepitaxial diamond layer.

As a conspicuous structure, hillocks were distributed even in etched sides in all samples. Cathode luminescence spectra measured at hillocks at etched sides are shown as (b) in Figs. 2 and 3. For sample \#01, the FE luminescence yield at the hillock was 1006 counts. No difference was found in comparison with the flat part. The electron irradiation area was ca. $190 \times 190 \mu \mathrm{m}$. The mean free path of the free exciton is expected to be a few micrometers. Therefore, few structural defects were found for the hillocks of sample \#01. The yield of FE luminescence of sample \#02 was 362 counts, which was approximately half that of the flat part. The crystalline quality at the hillock was not worse against impressions from their morphology. These hillocks are sometimes buried during crystal growth, sometimes becoming a source of abnormal growth.

In addition to etching treatment on a substrate, adoption of an off-angle controlled substrate is effective ${ }^{15)}$. As described above, individual differences of substrates strongly influence results. The off-angle of a substrate is expected to be an important part of the individual difference of a substrate. As the next step of study and development, influences of the off-angle of a substrate and etching treatment on a homoepitaxial diamond layer must be investigated in detail.

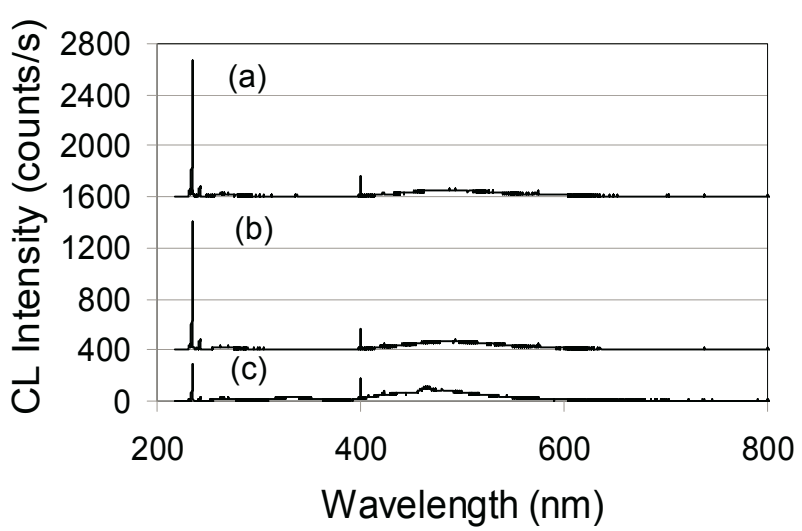

Fig.2 Cathode luminescence spectra obtained with sample \#01: a) flat surface on etched side, b) hillocks on etched side, c) flat surface on non etched side.

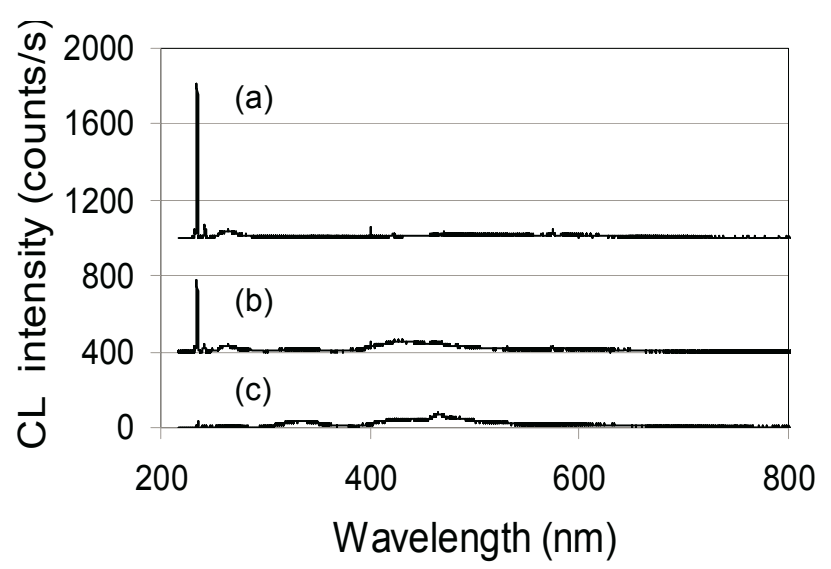

Fig.3 Cathode luminescence spectra obtained with sample \#02, a) flat surface on the etched side, b) hillocks on the etched side, c) flat surface on the non-etched side.

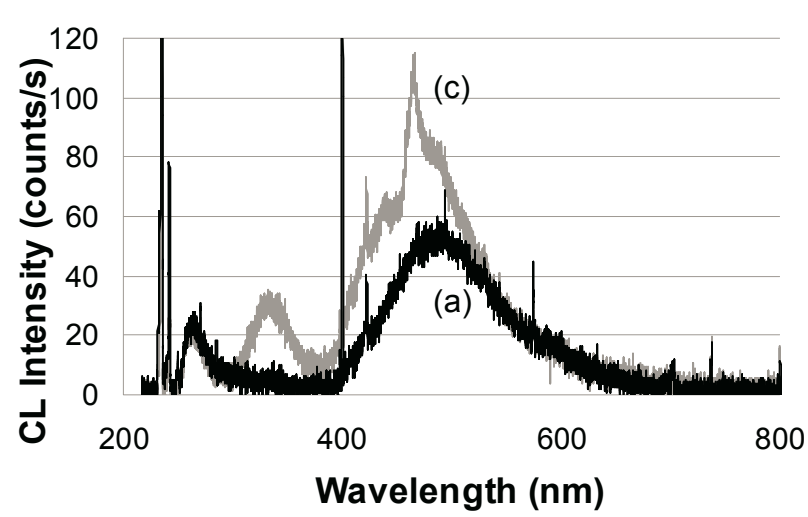

Fig.4 Close up of luminescence spectra obtained with sample \#01, a) flat surface on etched side, c) flat surface on non etched side.

\section{Acknowledgement}

A part of this study was supported by KAKENHI(20026 001). 


\section{References}

1) S.F.Kozlov, et al., IEEE Trans. Nucl. Sci.NS-22, 160(1975).

2) F.Nave, et al.,Nucl.Instr.and Meth. 26,308(1979).

3) S.F.Kozlov, et al.,IEEE Trans. Nucl. Sci. NS-24, 242(1977).

4) Krashlnikov, J. H. Kaneko, et al. Rev. Sci. Instrum.

5) N.Fujimori,S.Shikata,Frontier of Diamond for Electronics Application,214-228(2008), [in Japanese].

6) Michinori Yamamoto, Tokuyuki Teraji, Toshimichi Ito, "Improvement in the crystalline quality of homoepitaxial diamond films by oxygen plasma etching of mirror-polished diamond substrates", Journal of Crystal Growth 285 (2005) 130-136.

7) J.Vac.Sci.Technol.B3 (1), Jan/Feb,1985,p.146.

8) Lawrence S. Pan, DIAMOND: ELECTRONIC PRPERTIES AND APPLICATION, p.365.

9) N.Fujimori,S.Shikata, Frontier of Diamond for Electronics Application, 150-161(2008), [in Japanese].

10) T.Misu, M.Goto, T.Arai, ICPIG2003.

11) Dorsch, M. Werner, E. Obermeier, "Dry etching of undoped and boron doped polycrystalline diamond films", Diamond and Related Materials 4 (1995) 456-459.

12) Hiromu Shiomi, "Reactive Ion Etching of Diamond in $O 2$ and CF4 plasma, and Fabrication of Porous Diamond for Field Emitter Cathodes", Jpn. J. Appl. Phys. Vol. 36 (1997) pp. 7745-7748.

13) N.OhtakeM.Yoshikawa ,NEW DIAMOND, 87-89(2009).

14) N.Fujimori,S.Shikata, Frontier of Diamond for Electronics Application,214-228(2008), [in Japanese].

15) Norio Tokuda, Hitoshi Umezawa, Kikuo Yamabe, Hideyo Okushi, and Satoshi Yamazaki, "Hillock-Free Heavily Boron-DopedHomoepitaxial Diamond Films on Misoriented (001) Substrates", Japanese Journal of Applied Physics Vol.46, No47, (2007), pp1469-1470. 